\title{
Natriuretic peptide receptor A as a novel target for cancer
}

\author{
Jia Zhang, Zhilong Zhao and Jiansheng Wang*
}

\begin{abstract}
The receptor for the cardiac hormone atrial natriuretic peptide (ANP), natriuretic peptide receptor A (NPR-A), has been reported to be expressed in lung cancer, prostate cancer and ovarian cancer. NPR-A expression and signaling is important for tumor growth; its deficiency protects C57BL/6 mice from lung, skin and ovarian cancers. This suggests that NPR-A is a new marker and a new target for cancer therapy. Recently, NPR-A has been demonstrated to be expressed in pre-implantation embryos and in embryonic stem cells, which has a novel role in the maintenance of self-renewal and pluripotency of embryonic stem cells. A nanoparticle-formulated interfering RNA for NPR-A attenuated B16 melanoma tumors in mice. Ectopic expression of a plasmid encoding NP73-102, the NH2-terminal peptide of the ANP prohormone which downregulates NPR-A expression, also suppressed lung metastasis of A549 cells in nude mice and tumorigenesis of Line 1 cells in immunocompetent BALB/C mice. These results suggest that NPR-A is involved in tumorigenesis and a new target for cancer therapy. This review focuses on structure, abnormal functions and carcinogenic mechanisms of NPR-A to investigate its role in tumorigenesis.
\end{abstract}

Keywords: NPR-A, Cancer, Target

\section{Review}

\section{Introduction}

Natriuretic peptide receptor A (NPR-A) is the receptor for atrial natriuretic peptide (ANP) and brain natriuretic peptide (BNP). ANP and BNP, members of the natriuretic peptide family, have been extensively studied for their functions in regulating blood pressure. Both ANP and BNP signal through NPR-A by increasing cGMP and activating cGMP-dependent protein kinase G (PKG). Activated PKG in turn upregulates expression of genes encoding ion transporters and transcription factors, which together affect cell growth, apoptosis, proliferation, and inflammation [1-3]. The human NPR-A gene is located on chromosome 1q21-22 and consists of 22 exons and 21 introns within $16 \mathrm{~kb}$. NPR-A and/or its mRNA is expressed in kidney, lung, adipose, adrenal, brain, heart, testis, and vascular smooth muscle tissue [4-7]. Recent research suggests, however, that NPR-A expression and signaling is important for tumor growth. NPR-A deficiency protected C57BL/6 mice from lung, skin, and ovarian cancers. Furthermore, a nanoparticle-formulated interfering

\footnotetext{
* Correspondence: wangjshxjtu@gmail.com

Department of Thoracic Surgery 2, First Affiliated Hospital of Xi'an Jiaotong University, Xi'an, Shaanxi 710061, China
}

RNA for NPR-A attenuated B16 melanoma tumors in mice. Ectopic expression of a plasmid encoding NP73-102, the NH2-terminal peptide of the ANP prohormone which downregulates NPR-A expression, also suppressed lung metastasis of A549 cells in nude mice and tumorigenesis of Line 1 cells in immunocompetent BALB/c mice. These results suggest that NPR-A is involved in tumorigenesis and a new target for cancer therapy $[8,9]$. This review focuses on structure, abnormal functions and carcinogenic mechanisms of NPR-A to investigate its role in tumorigenesis.

\section{The structure of natriuretic peptide receptor $\mathrm{A}$}

The human NPR-A gene is approximately $16 \mathrm{~kb}$, contains 22 exons and 21 introns, and is located on chromosome 1q21-22 that encodes a 1,061-amino acid peptide [5,10]. The NPR-A gene product is abundantly expressed in the vascular system, kidneys, and adrenal glands [11]. NPR-A has been cloned and sequenced from rat brain $[12,13]$, human placenta [14], and mouse testis [15]. The general topological structure of NPR-A is consistent with that in the GC receptor family, containing at least four distinct regions: an extracellular ligand-binding domain (ECD), a single transmembrane spanning region, an intracellular protein kinase-like homology domain (protein-KHD), and 
a GC catalytic domain [15,16]. Activation of the NPR-A receptor requires ANP binding to the ECD and phosphorylation of up to six residues within the KHD [17]. After ANP binding to NPR-A, activation of the intrinsic GC leads to an intracellular increase in cGMP which in turn can activate different downstream effectors involved in cell growth, apoptosis, proliferation and inflammation, through the activation of cGMP-dependent PKG [3].

The ECD contains a highly conserved chloride-binding site near the ECD dimerization interface $[18,19]$. The ECD also contains in its juxtamembrane region a highly conserved structural motif, referred to as the receptor-GC signaling motif [20]. Single-residue mutations in this motif either render the receptor unresponsive to ligand binding or cause constitutive activation of GC activity [21], suggesting that this conserved structure plays a critical role in transmembrane signal transduction. The ECD contains three disulfide bonds, Cys60-Cys86, Cys164-Cys213 and Cys423-Cys432, in a 1-2, 3-4 and 5-6 linkage pattern [22]. Of these, the disulfide bond Cys60-Cys86 occurs in the chloride-binding site, and the disulfide bond Cys423Cys432 occurs in the juxtamembrane receptor-GC motif. Both disulfide bonds are conserved among the A-type and B-type natriuretic peptide receptors. The ECD also contains five $\mathrm{N}$-linked oligosaccharides [23]. Correct glycosylation is essential for expression of functional NPR-A: deletion of any one of the five glycosylation sites by mutagenesis reduces or abolishes NPR-A expression [24]. On the other hand, deglycosylation of the native or expressed ECD with endoglycosidase F2 or $\mathrm{H}$ has no effect on ANP binding [25]. Together, these findings suggest that glycosylation is essential for folding or transport of the nascent receptor polypeptide to the cell membrane, but that, once the active receptor is formed, the glycosyl moieties are not involved in ANP binding. This notion is consistent with the crystal structure of the ANP-ECD complex [18], which shows that glycosyl moieties or the glycosylation sites are located away from the ANP-binding site.

The KHD contains an approximately 280-amino acid region that immediately follows the transmembrane spanning domain of the receptor. The KHD of NPR-A is more closely related to protein tyrosine kinases than protein serine/threonine kinases. In fact, it is largely similar to the protein kinase domain of the platelet-derived growth factor receptor with approximately $31 \%$ amino acid sequence identity between the comparable regions of the kinase domains $[5,12,15]$. It has been demonstrated that the KHD of NPR-A serves an important mediatory role in transducing the ligand-induced signals to activate the GC catalytic domain of the receptor [16,26,27]. It has been suggested that an intervening step involving the KHD is necessary for the cyclase catalytic activation process $[24,28,29]$. Several studies have suggested that ATP serves as an intracellular allosteric regulator of the KHD for the activation of NPR-A [27,30-33]. ATP is thought to function by interacting with the KHD because this region contains a glycine-rich nucleotide-binding motif and has been postulated to provide an ATP-regulatory module for ANP signaling $[27,34]$. It has been also suggested that binding of ANP to NPR-A activates ATP binding to the KHD in the intracellular cytoplasmic space, which, in turn, activates the GC catalytic domain of the receptor $[27,28,35]$. Indeed, previous studies as well as recent data have indicated that the KHD seems to be important for ANP-dependent activation of NPR-A [36,37]. However, the exact mechanisms of activation and relay of signals from the KHD to the GC catalytic active site of the receptor remain to be established.

The GC catalytic domain of NPR-A contains an approximately 250-amino acid region that constitutes the catalytic active site of the receptor [38-40]. The transmembrane GC receptors contain a single cyclase catalytic active site per polypeptide molecule; however, based on the structure modeling data [41] two polypeptide chains seem to be required to activate NPR-A, and the receptor functions as a homodimer [42-44]. Initially, the dimerization region of the receptor was believed to be located between the KHD and GC domain that has been suggested to form an amphipathic alpha helix structure [45]. It has been reported that the crystal packing of the extracellular ligand-binding domain of NPR-A contains two possible dimer pairs, the head-to-head and tail-to-tail dimer pairs associated through the membrane-distal and membraneproximal subdomains, respectively [46]. The tail-to-tail dimer of NPR-A has been proposed previously [41]. The crystal structure of NPRC has also suggested that NPRC is dimerized in head-to-head configuration bound with ligand CNP [47]. It has been previously proposed that the head-to-head dimer may represent the latent inactive state and the tail-to-tail dimer could represent the hormone-activated state [48]. It has also been indicated that ligand-dependent activation of NPR-A stabilizes a membrane-distal dimer interface of this receptor protein suggesting that ANP binding stabilizes the NPR-A dimer with more stringent spacing at the dimer interface [49]. However, more recently, the results of site-directed mutagenesis and chemical modification studies suggested that head-to-head dimer structure reflects the physiological dimer structure of NPR-A [46].

\section{Natriuretic peptide receptor $A$ and cancer}

Almost all studies of NPR-A expression have focused on heart, kidney or other tissues. In 1993, Ohsaki and colleagues reported that functional ANP A receptors (NPR-A) are expressed in human small-cell lung cancer cell lines and Hela cells for the first time [50]. NPR-A mRNA was identified by Southern blot analyses of PCR products and RNase protection assays using poly (A) (+)-selected RNA. 
Vesely reported natriuretic peptides including ANP inhibit proliferation of various cancer cells and tumor growth, and NPR-A is expressed in various cancer cells [51], but he did not investigate the direct role of NPR-A in tumorigenesis. Recently the direct role of NPR-A signaling in tumorigenesis has been clarified by animal model, interfering RNA, Western blot and immunohistochemistry $[8,9]$.

\section{Natriuretic peptide receptor $A$ and lung cancer}

In 1993, NPR-A mRNA was identified by Southern blot analyses of PCR products and RNase protection assays using poly (A) (+)-selected RNA, and it was first reported expressed in human small-cell lung cancer cell lines [50]. Western blots results also show that NPR-A is present in human small-cell lung cancer cells [52]. Kong and colleagues [8] also found that NPR-A is expressed at a higher level in lung carcinoma (A549 and LLC1). They also clarified the direct role of NPR-A in lung cancer [8]. It has been shown NPR-A deficiency decreases lung inflammation. The data from animal models that compared the lungs of mice deficient in NPR-A (NPR-A ${ }^{-1-}$ ) with those of wild-type mice after immunization with intraperitoneal ovalbumin and subsequent challenge with intranasal ovalbumin showed that C57BL/6 wild-type mice had substantially higher inflammation, blocked airways, and goblet cell metaplasia than did NPR-A ${ }^{-/}$mice. Bronchoalveolar lavage fluid from $\mathrm{NPR}-\mathrm{A}^{-/-}$mice had significantly reduced levels of the inflammatory cytokines IL-4, IL-5, and IL-6 relative to those in wild-type mice. Using the Lewis lung carcinoma model, compared to $\mathrm{C} 57 \mathrm{BL} / 6$ wild-type mice, the tumor in NPR-A $\mathrm{A}^{-/-}$mice gradually shrank. This indicates that NPR-A deficiency significantly protects mice from lung tumorigenesis and lung tumor progression. NP73-102 nanoparticles were investigated in the suppression of lung cancer tumorigenesis [8]. Using a soft agar assay, A549 cells were transfected with pVAX1, pANP, or pNP73-102, and in vitro results from the soft agar assay indicated that cells transfected with pNP73-102 exhibited significantly decreased colony formation compared to that of nontransfected cells or cells transfected with pVAX1. Using a nude mouse model, mice were given $5 \times 10^{6}$ A549 cells intravenously and weekly instillations of PBS (control) or nanoparticles carrying pNP73-102 or pVAX1. Three weeks later, mice were sacrificed and lung sections were stained with hematoxylin and eosin and examined for lung nodules. Control animals receiving only PBS showed nodules and tumors, whereas the NP73-102treated group had no tumors. In the BALB/C mice tumor model, those treated with pNP73-102 nanoparticles injected weekly showed significantly reduced tumor burden compared to those treated with PBS or pVAX1 control vector [8]. TUNEL assay, Western blot of the cleavage of caspase 3 substrates, and PARP show that
NP73-102 induces apoptosis of A549 adenocarcinoma. Microarray analysis of gene expression of A549 cells after transfection with pNP73-102 show that pNP73-102 significantly altered the expression of a number of genes. The upregulated genes were predominantly from the family of interferon-regulated genes or related signal transduction pathways, and the downregulated genes included some involved in inflammation. This indicates that NP73-102 has anti-inflammatory properties.

To investigate the signal pathway in tumor suppression in NPR-A deficient mice, whole proteins were extracted from lungs of wild-type and NPR-A $\mathrm{A}^{-1-}$ mice and then probed using primary antibodies against p50, p65, phospho-p50, and phospho-p65. No significant difference in NF- $\mathrm{kB}$ expression in the lungs was abserved between wild-type and NPR-A $\mathrm{A}^{-1-}$ mice. However, the level of the activated form of NF- $\mathrm{B}$, phospho-NF- $\mathrm{B}$ (both phosphop65 and phospho-p50) was decreased in NPR-A $\mathrm{A}^{-/-}$mice. These results indicate that the role of NPR-A in lung inflammation may involve NF- $\mathrm{kB}$ activation. Immunohistochemistry analysis results from the expression of $\mathrm{pRb}$ in the lungs of wild-type $\mathrm{C} 57 \mathrm{BL} / 6$ and $\mathrm{NPR}-\mathrm{A}^{-/-}$mice revealed that NPR-A deficiency induced overexpression of pRb. Western blot results show that the expression of vascular endothelial growth factor was decreased in the lungs of NPR-A-deficient mice. Superarray analysis indicates that the expression of hexokinase 2, glycogen synthase 1 , and matrix metallopeptidase 10 were downregulated in the lungs of NPR-A $\mathrm{A}^{-/}$mice, while the expression of cellular retinol binding protein 1 was upregulated in the lungs of NPR-A ${ }^{-1-}$ mice.

\section{Natriuretic peptide receptor $A$ and skin cancer}

Kong and colleagues found that NPR-A is expressed at a higher level in melanoma [8]. siNPR-A nanoparticles were investigated in the injected melanoma cells wildtype mice model; the mice were treated twice a week with a cream containing either synthetic siNPR-A, psiNPR-A, or Scr, respectively. A significant reduction in tumor burden was seen in mice treated with siNPR-A (either with synthetic or psiNPR-A) but not those given Scr after 4 weeks [8]. This result indicates that siNPR-A could be used to treat melanomas. Flow cytometry assay results showed that overexpressing pNP73-102 in B16 melanoma could significantly induce apoptosis but not that of normal NIH3T3 cells [8]. TUNEL assay also showed a similar result.

\section{Natriuretic peptide receptor $A$ and ovarian cancer}

Kong and colleagues found that NPR-A is expressed at a higher level in ovarian cancer (SKOV3 and ID8) [8]. Using an animal model, wild-type and NPR-A-deficient C57BL/6 mice were injected with ovarian cancer cells and monitored weekly for tumor growth; by week 8 , all 
mice from the wild-type group developed solid tumors, but no tumors were observed in NPR-A-deficient mice [8]. This indicates NPR-A deficiency could inhibit the growth of ovarian cancer cells.

\section{Natriuretic peptide receptor $A$ and prostate cancer}

In 2005 Western blots revealed that for the first time NPR-A was present in prostate cancer cells [53]. Kong and colleagues found that NPR-A is expressed at a higher level in prostate cancer cells (DU145) [8]. Using Western blot and reverse transcriptase PCR, Wang and colleagues [9] demonstrated that NPR-A is abundantly expressed in tumorigenic mouse and human prostate cells, but not in non-tumorigenic prostate epithelial cells. Immunohistochemistry of tissue microarray showed NPR-A expression is positively correlated with clinical staging. siNPR-A or iNPR-A could induce apoptosis in Pca cells by the downregulation of NPR-A. This effect was linked to NPR-Ainduced expression of macrophage migration inhibitory factor (MIF), and it could be significantly reduced by siNPRA. Animal models show prostate tumor cells implanted into mice deficient in NPR-A failed to grow, and iNPR-A treatment could reduce the tumor burden and MIF expression in TRAMP-C1 xenografts. It was shown that NPR-A expression is correlated with MIF expression during PCa progression by the TRAMP spontaneous PCa model.

\section{Natriuretic peptide receptor $A$ and gastric cancer}

Our research mainly focuses on NPR-A and gastric cancer. NPR-A expression was examined by Western blot, immunofluorescence and immunohistochemistry. NPR-A was downregulated by transfection of NPR-A shRNA. We demonstrated that NPR-A is abundantly expressed in human gastric cancer AGS cells, but not in non-tumorigenic epithelial cells. NPR-A expression showed positive correlation with clinical staging and prognosis in gastric cancer patients. Downregulation of NPR-A by siNPR-A induced apoptosis in AGS cells. The mechanism of siNPR-Ainduced anti-AGS effects was linked to NPR-A-induced expression of KCNQ1, a $\mathrm{K}+$ channel overexpressed in AGS and significantly reduced by NPR-A shRNA.

\section{Natriuretic peptide receptor $A$ and other cancers}

NPR-A was also present in pancreatic adenocarcinoma cells [54], breast adenocarcinoma cells [55], colon adenocarcinoma cells [56], renal carcinoma cells [57], glioblastoma cells [58], and medullary thyroid carcinoma cells [59]. Vesely's group studied the effect of four peptides (long-acting natriuretic peptide, vessel dilator, kaliuretic peptide and atrial natriuretic peptide) on the cancer cells; they concluded that the mechanism of decrease in the number of cancer cells was via inhibition of DNA synthesis, mediated in part by cyclic GMP; they did not, however, investigate the role of NPR-A in cancer.
Carcinogenic mechanism of natriuretic peptide receptor A Athough Vesely's group only focused on the effect of natriuretic peptides (including ANP) on the proliferation of various cancer cells and tumor growth and they did not investigate the role of NPR-A in cancer, they showed that these peptides decrease expression of NPR-A [60]. The role of NPR-A in cancer has been clarified by Kong and colleagues using animal models and siRNA [8]. NPR-A-deficient mice showed significantly reduced antigen-induced pulmonary inflammation. NPR-A deficiency could protect C57BL/6 mice from lung, skin, and ovarian cancers. A nanoparticle formulated interfering RNA for NPR-A attenuated B16 melanoma tumors in mice. Ectopic expression of a plasmid encoding NP73-102 could suppress lung metastasis of A549 cells in nude mice and tumorigenesis of Line 1 cells in immunocompetent $\mathrm{BALB} / \mathrm{c}$ mice by the downregulation of NPR-A expression. Western blot and immunohistochemistry staining indicated that NF- $\mathrm{kB}$ was inactivated while retinoblastoma was upregulated in the lungs of NPR-A-deficient mice. The expression of vascular endothelial growth factor was also downregulated in the lungs of NPR-A-deficient mice compared with that in wild-type mice.

Recently it has been demonstrated that NPR-A is expressed in pre-implantation embryos and in embryonic stem cells. Knockdown of NPR-A by RNAi resulted in phenotypic changes, indicative of differentiation, downregulation of pluripotency factors and upregulation of differentiation genes. NPR-A knockdown also resulted in a marked downregulation of phosphorylated Akt [61]. These result from pre-implantation embryos and embryonic stem cells may give us some clues in the study of NPR-A in cancer.

\section{Conclusion}

Although NPR-A has been reported to be expressed in cancer cells for 30 years [50], it was not until 2008 that the role of NPR-A in cancer was studied [8]. Until now, only two papers have clarified the role of NPR-A in cancer $[8,9]$. Our research mainly focuses on the NPR-A and gastric cancer. We demonstrated that NPR-A is abundantly expressed on human gastric cancer AGS cells, but not in non-tumorigenic epithelial cells. NPR-A expression showed positive correlation with clinical staging and prognosis in gastric cancer patients. Downregulation of NPR-A by siNPR-A induced apoptosis in AGS cells. The mechanism of siNPR-A-induced antiAGS effects was linked to NPR-A-induced expression of KCNQ1, a $\mathrm{K}+$ channel overexpressed in AGS and significantly reduced by NPR-A shRNA. More research needs to focus on the role of NPR-A in cancer, which could clarify the carcinogenic mechanisms of NPR-A more clearly. 
From our results and others [8,9], it has been shown that NPR-A is a new target for cancer therapy. More research needs to focus on NPR-A to improve the efficiency of cancer therapy.

\section{Abbreviations}

ANP: atrial natriuretic peptide; BNP: brain natriuretic peptide; ECD: extracellular ligand-binding domain; IL: interleukin; KHD: kinase-like homology domain; MIF: migration inhibitory factor; NPR-A: natriuretic peptide receptor A; PBS: phosphate-buffered saline; PCR: polymerase chain reaction; PKG: protein kinase $\mathrm{G}$

\section{Competing interests}

The authors declare that they have no competing of interests.

\section{Authors' contributions}

JZ and ZZ wrote the paper. JW reviewed and edited the manuscript. All authors read and approved the final manuscript.

Received: 19 November 2013 Accepted: 9 May 2014

Published: 3 June 2014

\section{References}

1. Fiscus RR: Involvement of cyclic GMP and protein kinase $\mathrm{G}$ in the regulation of apoptosis and survival in neural cells. Neurosignals 2002, 11:175-190.

2. Pedram A, Razandi M, Kehrl J, Levin ER: Natriuretic peptides inhibit $G$ protein activation. Mediation through cross-talk between cyclic GMP-dependent protein kinase and regulators of $\mathrm{G}$ protein-signaling proteins. J Biol Chem 2000, 275:7365-7372.

3. Silberbach M, Roberts $C T$ Jr: Natriuretic peptide signalling: molecular and cellular pathways to growth regulation. Cell Signal 2001, 13:221-231.

4. Goy MF, Oliver PM, Purdy KE, Knowles JW, Fox JE, Mohler PJ, Qian X, Smithies O, Maeda N: Evidence for a novel natriuretic peptide receptor that prefers brain natriuretic peptide over atrial natriuretic peptide. Biochem J 2001, 358:379-387.

5. Lowe DG, Chang MS, Hellmiss R, Chen E, Singh S, Garbers DL, Goeddel DV: Human atrial natriuretic peptide receptor defines a new paradigm for second messenger signal transduction. EMBO J 1989, 8:1377-1384.

6. Nagase M, Katafuchi T, Hirose S, Fujita T: Tissue distribution and localization of natriuretic peptide receptor subtypes in stroke-prone spontaneously hypertensive rats. J Hypertens 1997, 15:1235-1243.

7. Wilcox JN, Augustine A, Goeddel DV, Lowe DG: Differential regional expression of three natriuretic peptide receptor genes within primate tissues. Mol Cell Biol 1991, 11:3454-3462.

8. Kong X, Wang X, Xu W, Behera S, Hellermann G, Kumar A, Lockey RF, Mohapatra S, Mohapatra SS: Natriuretic peptide receptor A as a novel anticancer target. Cancer Res 2008, 68:249-256.

9. Wang X, Raulji P, Mohapatra SS, Patel R, Hellermann G, Kong X, Vera PL, Meyer-Siegler KL, Coppola D, Mohapatra S: Natriuretic peptide receptor A as a novel target for prostate cancer. Mol Cancer 2011, 10:56.

10. Takahashi Y, Nakayama T, Soma M, Izumi Y, Kanmatsuse K: Organization of the human natriuretic peptide receptor A gene. Biochem Biophys Res Commun 1998, 246:736-739.

11. Garg R, Pandey KN: Regulation of guanylyl cyclase/natriuretic peptide receptor-A gene expression. Peptides 2005, 26:1009-1023.

12. Chinkers M, Garbers DL, Chang MS, Lowe DG, Chin HM, Goeddel DV, Schulz S: A membrane form of guanylate cyclase is an atrial natriuretic peptide receptor. Nature 1989, 338:78-83.

13. Schulz S, Singh S, Bellet RA, Singh G, Tubb DJ, Chin H, Garbers DL: The primary structure of a plasma membrane guanylate cyclase demonstrates diversity within this new receptor family. Cell 1989, 58:1155-1162.

14. Chang MS, Lowe DG, Lewis M, Hellmiss R, Chen E, Goeddel DV: Differential activation by atrial and brain natriuretic peptides of two different receptor guanylate cyclases. Nature 1989, 341:68-72.

15. Pandey KN, Singh S: Molecular cloning and expression of murine guanylate cyclase/atrial natriuretic factor receptor CDNA. J Biol Chem 1990, 265:12342-12348.
16. Garbers DL: Guanylyl cyclase receptors and their endocrine, paracrine, and autocrine ligands. Cell 1992, 71:1-4.

17. Pandey KN: Internalization and trafficking of guanylyl cyclase/natriuretic peptide receptor-A. Peptides 2005, 26:985-1000.

18. Ogawa H, Qiu Y, Ogata CM, Misono KS: Crystal structure of hormonebound atrial natriuretic peptide receptor extracellular domain: rotation mechanism for transmembrane signal transduction. J Biol Chem 2004, 279:28625-28631.

19. Ogawa H, Qiu Y, Philo JS, Arakawa T, Ogata CM, Misono KS: Reversibly bound chloride in the atrial natriuretic peptide receptor hormonebinding domain: possible allosteric regulation and a conserved structural motif for the chloride-binding site. Protein Sci 2010, 19:544-557.

20. Abe T, Misono KS: Proteolytic cleavage of atrial natriuretic factor receptor in bovine adrenal membranes by endogenous metalloendopeptidase. Effects on guanylate cyclase activity and ligand-binding specificity. Eur J Biochem 1992, 209:717-724.

21. Huo X, Abe T, Misono KS: Ligand binding-dependent limited proteolysis of the atrial natriuretic peptide receptor: juxtamembrane hinge structure essential for transmembrane signal transduction. Biochemistry 1999, 38:16941-16951.

22. Miyagi M, Misono KS: Disulfide bond structure of the atrial natriuretic peptide receptor extracellular domain: conserved disulfide bonds among guanylate cyclase-coupled receptors. Biochim Biophys Acta 2000, 1478:30-38.

23. Miyagi M, Zhang X, Misono KS: Glycosylation sites in the atrial natriuretic peptide receptor: oligosaccharide structures are not required for hormone binding. Eur J Biochem 2000, 267:5758-5768.

24. Koller KJ, Lipari MT, Goeddel DV: Proper glycosylation and phosphorylation of the type A natriuretic peptide receptor are required for hormonestimulated guanylyl cyclase activity. J Biol Chem 1993, 268:5997-6003.

25. Abe T, Nishiyama K, Snajdar R, He X, Misono KS: Aortic smooth muscle contains guanylate-cyclase-coupled 130-kDa atrial natriuretic factor receptor as predominant receptor form. Spontaneous switching to 60-kDa C-receptor upon cell culturing. Eur J Biochem 1993, 217:295-304.

26. Chinkers M, Garbers DL: The protein kinase domain of the ANP receptor is required for signaling. Science 1989, 245:1392-1394.

27. Duda T, Goraczniak RM, Sharma RK: Core sequence of ATP regulatory module in receptor guanylate cyclases. FEBS Lett 1993, 315:143-148.

28. Goraczniak RM, Duda T, Sharma RK: A structural motif that defines the ATP-regulatory module of guanylate cyclase in atrial natriuretic factor signalling. Biochem J 1992, 282:533-537.

29. Koller KJ, de Sauvage FJ, Lowe DG, Goeddel DV: Conservation of the kinase-like regulatory domain is essential for activation of the natriuretic peptide receptor guanylyl cyclases. Mol Cell Biol 1992, 12:2581-2590.

30. Chinkers $M$, Singh $S$, Garbers DL: Adenine nucleotides are required for activation of rat atrial natriuretic peptide receptor/guanylyl cyclase expressed in a baculovirus system. J Biol Chem 1991, 266:4088-4093.

31. Kurose $H$, Inagami T, Ui M: Participation of adenosine $5^{\prime}$-triphosphate in the activation of membrane-bound guanylate cyclase by the atrial natriuretic factor. FEBS Lett 1987, 219:375-379.

32. Larose L, McNicoll N, Ong H, De Lean A: Allosteric modulation by ATP of the bovine adrenal natriuretic factor R1 receptor functions. Biochemistry 1991, 30:8990-8995.

33. Wong SK, Ma CP, Foster DC, Chen AY, Garbers DL: The guanylyl cyclase-A receptor transduces an atrial natriuretic peptide/ATP activation signal in the absence of other proteins. J Biol Chem 1995, 270:30818-30822

34. Sharma RK: Evolution of the membrane guanylate cyclase transduction system. Mol Cell Biochem 2002, 230:3-30.

35. Jewett JR, Koller KJ, Goeddel DV, Lowe DG: Hormonal induction of low affinity receptor guanylyl cyclase. EMBO J 1993, 12:769-777.

36. Larose L, Rondeau JJ, Ong H, De Lean A: Phosphorylation of atrial natriuretic factor $\mathrm{R} 1$ receptor by serine/threonine protein kinases: evidences for receptor regulation. Mol Cell Biochem 1992, 115:203-221.

37. Potter $L R$, Hunter $T$ : Identification and characterization of the major phosphorylation sites of the B-type natriuretic peptide receptor. J Biol Chem 1998, 273:15533-15539.

38. Liu Y, Ruoho AE, Rao VD, Hurley JH: Catalytic mechanism of the adenylyl and guanylyl cyclases: modeling and mutational analysis. Proc Natl Acad Sci U S A 1997, 94:13414-13419.

39. Sunahara RK, Beuve A, Tesmer JJ, Sprang SR, Garbers DL, Gilman AG: Exchange of substrate and inhibitor specificities between adenylyl and guanylyl cyclases. J Biol Chem 1998, 273:16332-16338. 
40. Tucker $\mathrm{CL}$, Hurley JH, Miller TR, Hurley JB: Two amino acid substitutions convert a guanylyl cyclase, RetGC-1, into an adenylyl cyclase. Proc Natl Acad Sci U S A 1998, 95:5993-5997.

41. van den Akker F, Zhang X, Miyagi M, Huo X, Misono KS, Yee VC: Structure of the dimerized hormone-binding domain of a guanylylcyclase-coupled receptor. Nature 2000, 406:101-104

42. Labrecque J, Mc Nicoll N, Marquis M, De Lean A: A disulfide-bridged mutant of natriuretic peptide receptor-A displays constitutive activity. Role of receptor dimerization in signal transduction. J Biol Chem 1999, 274:9752-9759.

43. Wilson EM, Chinkers M: Identification of sequences mediating guanylyl cyclase dimerization. Biochemistry 1995, 34:4696-4701.

44. Yang RB, Garbers DL: Two eye guanylyl cyclases are expressed in the same photoreceptor cells and form homomers in preference to heteromers. J Biol Chem 1997, 272:13738-13742.

45. Garbers DL, Lowe DG: Guanylyl cyclase receptors. J Biol Chem 1994, 269:30741-30744.

46. Qiu Y, Ogawa H, Miyagi M, Misono KS: Constitutive activation and uncoupling of the atrial natriuretic peptide receptor by mutations at the dimer interface. Role of the dimer structure in signalling. $J$ Biol Chem 2004, 279:6115-6123.

47. He X, Chow D, Martick MM, Garcia KC: Allosteric activation of a spring-loaded natriuretic peptide receptor dimer by hormone. Science 2001, 293:1657-1662.

48. van den Akker F: Structural insights into the ligand binding domains of membrane bound guanylyl cyclases and natriuretic peptide receptors. J Mol Biol 2001, 311:923-937.

49. De Lean A, McNicoll N, Labrecque J: Natriuretic peptide receptor A activation stabilizes a membrane-distal dimer interface. J Biol Chem 2003, 278:11159-11166.

50. Ohsaki Y, Yang HK, Le PT, Jensen RT, Johnson BE: Human small cell lung cancer cell lines express functional atrial natriuretic peptide receptors. Cancer Res 1993, 53:3165-3171.

51. Vesely DL: Atrial natriuretic peptides: anticancer agents. J Investig Med 2005, 53:360-365.

52. Vesely BA, Song S, Sanchez-Ramos J, Fitz SR, Alli AA, Solivan SM, Gower WR $\mathrm{Jr}$, Vesely DL: Five cardiac hormones decrease the number of human small-cell lung cancer cells. Eur J Clin Invest 2005, 35:388-398.

53. Vesely BA, Alli AA, Song SJ, Gower WR Jr, Sanchez-Ramos J, Vesely DL: Four peptide hormones specific decrease (up to 97\%) of human prostate carcinoma cells. Eur J Clin Invest 2005, 35:700-710.

54. Vesely BA, McAfee Q, Gower WR Jr, Vesely DL: Four peptides decrease the number of human pancreatic adenocarcinoma cells. Eur J Clin Invest 2003 33:998-1005.

55. Vesely BA, Song S, Sanchez-Ramos J, Fitz SR, Solivan SM, Gower WR Jr, Vesely DL: Four peptide hormones decrease the number of human breast adenocarcinoma cells. Eur J Clin Invest 2005, 35:60-69.

56. Gower WR, Vesely BA, Alli AA, Vesely DL: Four peptides decrease human colon adenocarcinoma cell number and DNA synthesis via cyclic GMP. Int J Gastrointest Cancer 2005, 36:77-87.

57. Vesely BA, Eichelbaum EJ, Alli AA, Sun Y, Gower WR Jr, Vesely DL: Urodilatin and four cardiac hormones decrease human renal carcinoma cell numbers. Eur J Clin Invest 2006, 36:810-819.

58. Vesely BA, Eichelbaum EJ, Alli AA, Sun Y, Gower WR Jr, Vesely DL: Four cardiac hormones eliminate 4-fold more human glioblastoma cells than the green mamba snake peptide. Cancer Lett 2007, 254:94-101.

59. Eichelbaum EJ, Vesely BA, Alli AA, Sun Y, Gower WR Jr, Vesely DL: Four cardiac hormones eliminate up to $82 \%$ of human medullary thyroid carcinoma cells within 24 hours. Endocrine 2006, 30:325-332.

60. Vesely DL: Cardiac and renal hormones: anticancer effects in vitro and in vivo. J Investig Med 2009, 57:22-28.

61. Abdelalim EM, Tooyama I: NPR-A regulates self-renewal and pluripotency of embryonic stem cells. Cell Death Dis 2011, 2:e127.

doi:10.1186/1477-7819-12-174

Cite this article as: Zhang et al:: Natriuretic peptide receptor $\mathrm{A}$ as a

novel target for cancer. World Journal of Surgical Oncology 2014 12:174.

\section{Submit your next manuscript to BioMed Central and take full advantage of:}

- Convenient online submission

- Thorough peer review

- No space constraints or color figure charges

- Immediate publication on acceptance

- Inclusion in PubMed, CAS, Scopus and Google Scholar

- Research which is freely available for redistribution

Submit your manuscript at www.biomedcentral.com/submit
C) Biomed Central 